\title{
Structural basis of mRNA cap modification by SARS-CoV-2: role of metal ions and implications for COVID-19 severity by emerging variants
}

\author{
Y. K. Gupta ${ }^{1}$, T. Viswanathan ${ }^{1}$, A. Misra ${ }^{1}$, S-H. Chan ${ }^{2}$, S. Qi ${ }^{1}$, S. Arya ${ }^{1}$, N. Dai ${ }^{2}$, L. Martinez-Sobrido ${ }^{3}$, \\ ${ }^{1}$ University of Texas Health at San Antonio, San Antonio, USA, \\ ${ }^{2}$ New England Biolabs, Ipswich, MA, USA, ${ }^{3}$ Texas Biomedical Research Institute, San Antonio, USA
}

guptay@uthscsa.edu

The severe acute respiratory syndrome coronavirus-2 (SARS-CoV-2), the causative agent of COVID-19 illness is responsible for more than half a million deaths in the United States alone. The SARS-CoV-2 nsp16/nsp10 enzyme complex modifies the 2'-OH of the first transcribed nucleotide $\left(\mathrm{N}_{1}\right.$ base) of the viral mRNA by covalently attaching a methyl group to it. This single RNA modification event converts the status of the mRNA cap from Cap- $0\left({ }^{\mathrm{m} 7} \mathrm{GpppA}\right)$ to Cap- $1\left({ }^{\mathrm{m} 7} \mathrm{GpppAm}\right)$ and helps the virus evade immune surveillance in the host cell. Here, we report three high-resolution crystal structures of nsp16/nsp10 heterodimer representing substrate (Cap-0)-bound state, and pre- and post-release states of the RNA product (Cap-1). The binding of Cap-0 induces large conformational changes. This 'induced fit' model provides new mechanistic insights into the 2'-O methylation of the viral mRNA cap. We reveal the structural basis for the RNA specificity of nsp16/nsp10. We also discover an alternative ligand-binding site unique to SARS-CoV-2 [1]. We also observe overall widening of the enzyme upon product formation, and an inward twisting motion in the substrate-binding region upon product release. These changes reset the enzyme for the next round of catalysis, and may be the structural basis of dissociation nsp10 from nsp16. The structures also identify a unique binding mode of a divalent metal ion in nsp16, which aligns the first two bases of the viral RNA in the catalytic pocket for efficient Cap-1 formation. Using LC/MS-based intact mass analysis, we show dramatic perturbations in Cap-1 formation by an emerging clinical variant of SARS-CoV-2, previous SARS-CoV outbreak strain, and their altered sensitivity to divalent metal ions [2]. Such reliance and preference for metals also suggests that an imbalance in cellular metal concentrations could differentially alter the RNA capping and thus, host innate immune response to infections by various CoVs. Altogether, our work provides a revised framework from which new therapeutic modalities may be designed for the treatment of COVID-19 and emerging coronavirus illnesses.

[1] Viswanathan, T., Arya, S., Chan, S. H., Qi, S., Dai, N., Misra, A., Park, J. G., Oladunni, F., Kovalskyy, D., Hromas, R. A., Martinez-Sobrido, L., and Gupta, Y. K. (2020) Structural basis of RNA cap modification by SARS-CoV-2, Nat Commun 11, 3718.

[2] Viswanathan, T., Misra, A., Chan, S. H., Qi, S., Dai, N., Arya, S., Martinez-Sobrido, L., and Gupta, Y. K. (2021) A metal ion orients mRNA to ensure accurate 2'- O methylation of its first nucleotide, Nat Commun 12(1):3287

\section{Keywords: SARS-CoV-2; RNA capping; mRNA modification; SAM-dependent methyltransferases; X-ray crystallography}

Authors thank the UT Health San Antonio, Voelcker Foundation, San Antonio Partnership for Precision Therapeutics, Institute of Integration for Medicine and Science, Greehey Children's Cancer Research Institute, San Antonio Area Foundation, and Cancer Prevention Research Institute of Texas for their support. 